\title{
O ensino da física térmica e o forno solar: uma revisão
}

\section{Teaching thermal physics and the solar oven: A review}

Jonas Cegelka da Silva1; Isabel Krey Garcia 2; Luiz Caldeira Brant de Tolentino Neto $^{3}$

\section{RESUMO}

Fazer uma busca bibliográfica sobre determinado assunto é um passo importante no desenvolvimento de pesquisas, uma vez que coloca o pesquisador em contato com os materiais já publicados sobre o tema. A busca bibliográfica realizada neste trabalho abrangeu anais de eventos, periódicos científicos, teses e dissertações no período compreendido entre 2005 a 2015, resultando em um total de 104 trabalhos pré-selecionados. Foi realizada uma leitura exploratória desse material e 32 foram definidos para uma leitura analítica, especificamente aqueles relacionados ao ensino médio. Dentre os resultados da revisão, pode-se destacar um grande número de pesquisas voltadas às metodologias de ensino que facilitam a compreensão dos conceitos da física térmica; a importância de trabalhos nessa área em função da confusão conceitual existente entre os conceitos de calor e temperatura e; o forno solar aparece em diversos trabalhos como um dispositivo potencial para abordar os conceitos relativos à física térmica, o que demonstra a conexão apontada no título do artigo.

Palavras-chave: calor, temperatura, interdisciplinaridade, forno solar.

\begin{abstract}
Searching the literature for a given subject is an important step for research papers, putting the researcher in contact with the sources already published on the issue. In this study, this quest included papers published in scientific events, scientific journals, dissertations, and theses released between 2005 and 2015, totalizing 104 pre-selected papers. We performed an exploratory reading of these documents, selecting 32 for further analytical reading, giving preference to those related to high-school audience. Among the findings, we encountered a large number of studies focused on teaching methods for thermal physics concepts. Research in this area should be undertaken since there is a confusion between the concepts of heat and temperature. Solar oven has been cited in several papers as a potential device to approach the concepts related to thermal physics, as pointed out in the title of this paper.
\end{abstract}

Keywords: heat, temperature, interdisciplinarity, solar oven.

\footnotetext{
${ }^{1}$ IFFARROUPILHA - Instituto Federal de Educação, Ciência e Tecnologia Farroupilha, Santa Rosa/RS - Brasil.

2 UFSM - Universidade Federal de Santa Maria, Santa Maria/RS - Brasil.

3 UFSM - Universidade Federal de Santa Maria, Santa Maria/RS - Brasil.
} 


\section{INTRODUÇÃO}

A construção histórica do conceito de calor evoluiu desde a teoria do flogisto até o princípio da conservação da energia e do equivalente mecânico do calor. No entanto, os conceitos de calor e temperatura são entendidos, corriqueiramente, como sendo sinônimos ou dependentes e essa visão repete-se na escola, espaço no qual os alunos têm dificuldade em entender as diferenças existentes. Essas concepções alternativas dos alunos podem também ser induzidas pelos livros didáticos (GOMES, 2012). Sobre essa contradição na relação entre os conceitos,

A teoria do calórico e a ideia do calor como oposição ao frio, como entidade física, ideias há muito desconstruídas pela física, ainda persistem no senso comum porque são suficientes para a vida diária. Continuamos a falar que nossos casacos nos "protegem" do frio, que devemos fechar a geladeira porque senão o "frio" sai (Lopes, 1999, p. 143).

Muitas vezes, calor é exprimido de forma a induzir que corresponde a uma propriedade intrínseca de um corpo, ao ponto que o corpo pode "ganhá-lo" ou "perdê-lo". Ao contrário disso, "a transferência de energia produzida apenas por uma diferença de temperatura denomina-se transferência de calor ou fluxo de calor, e a energia transferida desse modo denomina-se calor" (Young \& Freedman, 2009, p. 190).

Já a temperatura está relacionada com a visão quantitativa de quente ou frio e à energia cinética das moléculas de um corpo. Dessas conceituações, é possível perceber que os conceitos não são sinônimos, uma vez que, ao contrário da temperatura, o calor não é uma propriedade intrínseca de um sistema, tendo significado apenas quando descreve a transferência de energia para dentro ou fora do sistema (Halliday, et al., 2009, p.190).

Ensinar esses conceitos, especialmente no ensino médio, é uma tarefa que requer a exploração de vieses históricos e experimentais, como forma de enriquecer o estudo e possibilitar que a aprendizagem ocorra de forma significativa (à luz da teoria de Ausubel). E um recurso facilitador para essas abordagens é a revisão bibliográfica sobre $o$ assunto, fonte nas quais se encontram pesquisas desenvolvidas com aspectos similares ou até mesmo motivadores para a prática docente. Nesse sentido, de acordo com Laville \& Dionne (1999), fazer a revisão da literatura significa tentar encontrar as pesquisas já realizadas sobre o tema para, a partir delas, aperfeiçoar nosso conhecimento, afinar nossa perspectiva teórica, além de tornar mais consciente e articulada nossas intenções a partir da análise de como outros procederam em suas pesquisas.

Para este escrito, a busca bibliográfica objetivou levantar trabalhos desenvolvidos com estudantes do ensino médio (público para o qual atuamos) que investigaram a construção dos conceitos da calorimetria. Centralizamos nossa busca naqueles trabalhos que se fundamentam na teoria da aprendizagem significativa, na teoria dos campos conceituais e na interdisciplinaridade, que são teorias que fazem parte do nosso fazer pedagógico. $\mathrm{O}$ forno solar insere-se na busca pelo fato de ser um recurso didático interessante para abarcar os conceitos a serem estudados, uma vez que seu princípio de funcionamento está relacionado à elevação da temperatura em seu interior, dependendo da escolha dos materiais utilizados e da eficácia do isolamento para evitar as trocas térmicas.

De acordo com Ausubel (2003, p. 71), a essência do processo da aprendizagem significativa consiste no fato de que as novas ideias a serem aprendidas devem se relacionam com aquilo que o aluno já 
sabe (subsunçores) de forma não arbitrária e não literal. Para que ocorra aprendizagem significativa, duas condições devem ser satisfeitas: (i) o material a ser ensinado deve ser potencialmente significativo, ou seja, deve estar relacionado de forma plausível, sensível, não aleatória e não literal com qualquer estrutura cognitiva apropriada e relevante e; (ii) o aluno deve estar predisposto a aprender.

A este processo de atribuição de novos significados a um determinado subsunçor, Ausubel chama de diferenciação progressiva que consiste em reconhecer que as ideias, conceitos ou proposições mais gerais e inclusivos de determinado conteúdo devem ser apresentados primeiro e, progressivamente diferenciados em termos de detalhes e especificidade. Conforme o sujeito modifica seus subsunçores, ocorre simultaneamente o princípio da reconciliação integrativa, a qual consiste em explorar relações entre ideias, eliminar diferenças aparentes, resolver inconsistências e integrar significados (Moreira, 1999).

Ponderando as implicações desta teoria para o ensino-aprendizagem, a variável isolada mais importante para a aprendizagem significativa de novos conhecimentos é aquilo que o aluno já sabe os subsunçores existentes na sua estrutura cognitiva (Ausubel, 2003). Isso indica que é necessário conhecer a bagagem conceitual dos alunos para, a partir dessa, criar roteiros e estratégias didáticas que permitam a eles evoluírem em um determinado campo conceitual.

Campo conceitual, de acordo com Vergnaud (1986), corresponde a um conjunto de situações cujo domínio requer uma variedade de conceitos, de procedimentos e de representações simbólicas em estreita conexão, de modo que (i) um conceito não se forma com um só tipo de situação; (ii) a análise de uma situação requer a utilização de vários conceitos e; (iii) a formação de um conceito demora um período longo de tempo.

Um conceito (C) refere-se a um conjunto de situações (S), compreendendo um conjunto de diferentes invariantes operatórios (teoremas-em-ação e conceitos-em-ação) (I), e suas propriedades podem ser expressas por diferentes representações simbólicas $(R)$, que pode ser esquematizado da forma $C=$ (S, I, R). Se os conceitos tornam-se significativos através de diversas situações (no sentido de tarefa ou problema), estas são a principal entrada de um campo conceitual. $E$, no processo de ensinoaprendizagem, a primeira ação de mediação dos professores é, de fato, a seleção das situações que serão propostas.

Um teorema-em-ação corresponde a uma proposição que pode ser verdadeira ou falsa, enquanto um conceito-em-ação (objeto ou predicado) é ou não é relevante. Acontece que no processo de conceitualização do real, esses invariantes operatórios envolvidos na organização de atividades frequentemente permanecem implícitos ou mesmo inconscientes (Vergnaud, 2013). O professor deve, então, procurar os conceitos-em-ação e teoremas-em-ação utilizados pelos alunos, identificando se são suficientes para responder às novas situações apresentadas ou ainda se estão servindo de obstáculo cognitivo para a nova aprendizagem.

A interdisciplinaridade, nesse sentido, converge com a teoria da aprendizagem significativa de Ausubel e com a teoria dos campos conceituais de Vergnaud na medida em que traz uma perspectiva que visa romper com o currículo estanque, fragmentado e rígido que acontece na maioria das escolas. Para Fazenda (2008), pensar a interdisciplinaridade requer o favorecimento do processo de aprendizagem, respeitando os saberes dos alunos e sua integração. 
Faz-se interdisciplinaridade, de acordo com Pombo (2013), quando se consegue fazer uma combinação, uma convergência ou uma complementaridade, objetivando a compreensão de um objeto a partir de pontos de vista diferentes, implicando uma reorganização dos processos de ensino. Deve haver também, conforme Fazenda (2011), um regime de copropriedade que implica o diálogo entre os professores envolvidos, de modo a se ter uma intensa reciprocidade nas trocas, visando a um enriquecimento mútuo e eliminando as barreiras entre as disciplinas e entre as pessoas.

A partir dessas considerações, o escrito em tela caracteriza-se pela revisão bibliográfica sobre a física térmica e o forno solar, em diferentes fontes, focando no percurso metodológico das pesquisas realizadas, na busca de atividades diferenciadas sobre os conceitos trabalhados e diferentes abordagens sobre interdisciplinaridade. Os materiais já publicados sobre o ensino da física térmica tornam-se potenciais para enriquecer nossa prática de sala de aula, bem como servir de base para outros trabalhos investigativos sobre o tema.

\section{MATERIAIS E MÉTODOS}

A busca bibliográfica abrangeu anais de eventos, periódicos científicos, teses e dissertações no período entre 2005 a 2015, que estavam disponíveis on-line e compreendeu um trabalho em três fases como apontam Markoni \& Lakatos (2011): identificação (seleção dos materiais a serem pesquisados); compilação (agrupamento sistemático do material); e fichamento (transcrição dos principais dados encontrados às fichas bibliográficas).

As palavras-chave definidas para a busca foram: calorimetria, conforto térmico, energia solar, física térmica, fogão/forno solar, termodinâmica, termologia e termometria, selecionando apenas aqueles trabalhos voltados ao ensino. Os periódicos escolhidos para análise foram: Aprendizagem Significativa em Revista (ASR), Caderno Brasileiro de Ensino de Física (CBEF), Experiências em Ensino de Ciências (EENCI), Latin-American Journal of Physics Education (LAJPE), Revista Brasileira de Ensino de Física (RBEF), Ciência \& Educação (CE), Revista Electrónica de Enseñanza de las Ciencias (REEC), Enseñanza de las Ciencias (ELC) e Investigação em Ensino de Ciências (IENCI). Esses periódicos foram escolhidos porque têm disponibilidade on-line (e gratuita), significativa relevância para a área do ensino, além de serem bem avaliadas no Qualis na área de ensino da Capesi, na avaliação dos periódicos de 2015, como mostrado no Quadro 1.

Se analisarmos os Qualis 2015 da Capes, temos 122 periódicos classificados como A1 e 147 classificados como A2 na área de ensino, totalizando 269 periódicos com as melhores avaliações, tanto nacionais como internacionais. Nossa escolha se deu pelos 9 citados acima, pois têm uma representatividade nacional e estrangeira, dando uma amostra dos trabalhos publicados.

\begin{tabular}{|l|c|}
\hline \multicolumn{1}{|c|}{ Periódico } & Qualis Ensino (2015) \\
\hline Aprendizagem Significativa em Revista & B3 \\
\hline Caderno Brasileiro de Ensino de Física & A2 \\
\hline Ciência \& Educação & A1 \\
\hline Enseñanza de las Ciencias & A1 \\
\hline Experiências em Ensino de Ciências & B1 \\
\hline Investigação em Ensino de Ciências & A2 \\
\hline
\end{tabular}




\begin{tabular}{|l|l|}
\hline Latin-American Journal of Physics Education & B1 \\
\hline Revista Brasileira de Ensino de Física & A1 \\
\hline Revista Electrónica de Enseñanza de las Ciencias & A2 \\
\hline
\end{tabular}

Quadro 1. Avaliação Qualis da Capes para periódicos em 2015.

Para busca nos periódicos ASR, IENCI, EENCI, REEC e LAJPE, as edições desses foram abertas individualmente e os artigos de interesse foram avaliados, inicialmente, a partir do título e das palavras-chave. A seleção dos artigos do CBEF e ELC ocorreu diretamente na página dos periódicos, nas quais as palavras-chave foram inseridas no espaço "pesquisa" do campo "conteúdo da revista", considerando todos os escopos de busca (título, autor, resumo, texto completo). Já a escolha dos artigos da RBEF e da CE se deu na página da biblioteca eletrônica Scielo, na qual as palavras-chave foram inseridas no espaço "pesquisa", considerando todos os escopos de busca (título, autor, assunto).

A partir da delimitação das palavras-chave, foram pré-selecionados 41 artigos publicados nos periódicos. Foi realizada uma leitura exploratória dessas obras, com o objetivo de verificar se convergiam com nossos objetivos (GIL, 2002) e 11 deles foram selecionados para uma leitura analítica. O Quadro 2 mostra esses textos com o local e ano de publicação, além de sua autoria.

\begin{tabular}{|l|l|c|c|}
\hline \multicolumn{1}{|c|}{ Autor } & \multicolumn{1}{|c|}{ Título } & Ano & Publicação \\
\hline Damasio \& Steffani & $\begin{array}{l}\text { Ensinando física com consciência ecológica e com } \\
\text { materiais descartáveis }\end{array}$ & 2007 & RBEF \\
\hline $\begin{array}{l}\text { Carvalho Júnior \& } \\
\text { Aguiar Júnior }\end{array}$ & $\begin{array}{l}\text { Os campos conceituais de Vergnaud como ferramenta } \\
\text { para o planejamento didático }\end{array}$ & 2008 & CBEF \\
\hline Grings, et al. & $\begin{array}{l}\text { Avanços e retrocessos dos alunos no campo conceitual } \\
\text { da Termodinâmica }\end{array}$ & 2008 & REEC \\
\hline Silva, et al. & $\begin{array}{l}\text { Reflexões para subsidiar discussões sobreo conceito } \\
\text { de calor na sala de aula }\end{array}$ & 2008 & CBEF \\
\hline Dorado \& Rivera & $\begin{array}{l}\text { Enseñanza de las ciencias físicas a estudiantes de } \\
\text { primaria y secundaria por medio de sencillos talleres } \\
\text { científicos }\end{array}$ & 2010 & LAJPE \\
\hline Dworakowski, et al. & O aquecedor solar na sala de aula & 2010 & EENCI \\
\hline Gomes & A ascenção e queda da teoria do calórico & 2012 & CBEF \\
\hline Hernández \& Dávalos & $\begin{array}{l}\text { La Termodinámica como origen de la revolución } \\
\text { industrial del siglo XVIII }\end{array}$ & 2012 & LAJPE \\
\hline Ramos, et al. & $\begin{array}{l}\text { An alternative for the teaching and learning of the heat } \\
\text { transmission topic with base in the directed research } \\
\text { for high-school students }\end{array}$ & 2012 & LAJPE \\
\hline Pérez & $\begin{array}{l}\text { Perspectivas didácticas de una aproximación histórica } \\
\text { a la medición y concepto de Temperatura }\end{array}$ & 2013 & LAJPE \\
\hline Silva, et al. & $\begin{array}{l}\text { Concepções sobre a natureza do calor em diferentes } \\
\text { contextos históricos }\end{array}$ & 2013 & CBEF \\
\hline
\end{tabular}

Quadro 2. Artigos analisados dos periódicos científicos.

As teses e dissertações ${ }^{i i}$ foram obtidas na Biblioteca Digital Brasileira de Teses e Dissertações. No portal, foi feita a busca considerando todos os campos (título, autor, assunto, instituição) e um total de 40 trabalhos foi pré-selecionado. Também, depois de uma leitura exploratória, foi realizada a 
leitura analítica dos 11 escolhidos por se encaixarem nos nossos objetivos de revisão. O Quadro 3 mostra esses trabalhos com o local e ano de publicação, além de sua autoria.

\begin{tabular}{|c|c|c|c|}
\hline Autor & Título & Ano & Publicação \\
\hline Sias & $\begin{array}{l}\text { A aquisição automática de dados proporcionando discussões } \\
\text { conceituais na física térmica do ensino médio }\end{array}$ & 2006 & Dissertação \\
\hline Rafael & $\begin{array}{l}\text { Elaboração e aplicação de uma estratégia de ensino sobre os } \\
\text { conceitos de calor e de temperatura }\end{array}$ & 2007 & Dissertação \\
\hline Silva & $\begin{array}{l}\text { Mapas conceituais como instrumentos de promoção e } \\
\text { avaliação da aprendizagem significativa de conceitos de } \\
\text { calorimetria, em nível médio }\end{array}$ & 2007 & Dissertação \\
\hline Michelena & Física térmica: uma abordagem histórica e experimental & 2008 & Dissertação \\
\hline Amaral & $\begin{array}{l}\text { A atividade prática abordada por meio de situações- } \\
\text { problema, visando a promoção da aprendizagem } \\
\text { significativa dos conceitos relacionados aos processos de } \\
\text { transmissão da energia térmica, no ensino médio }\end{array}$ & 2010 & Dissertação \\
\hline Dal Moro & $\begin{array}{l}\text { Do trabalho para a escola: olhares de trabalhadores- } \\
\text { estudantes e professores sobre as relações entre o saber da } \\
\text { prática e o saber da escola }\end{array}$ & 2012 & Dissertação \\
\hline Oliveira & $\begin{array}{l}\text { Atividade experimental investigativa: construção do } \\
\text { termômetro de coluna líquida }\end{array}$ & 2013 & Dissertação \\
\hline Poglia & $\begin{array}{l}\text { O refrigerador doméstico como instrumento motivador para } \\
\text { o ensino de física térmica: uma proposta para o curso } \\
\text { Técnico Integrado em Refrigeração e Climatização }\end{array}$ & 2013 & Dissertação \\
\hline Pradella & $\begin{array}{l}\text { Estudo de conceitos da termodinâmica no ensino médio por } \\
\text { meio de UEPS }\end{array}$ & 2014 & Dissertação \\
\hline Pereira & $\begin{array}{l}\text { Memória mediada na aprendizagem de Física: } \\
\text { problematizando a afirmaação "Não me lembro de nada das } \\
\text { aulas do ano passado!" }\end{array}$ & 2014 & Tese \\
\hline Faccin & $\begin{array}{l}\text { Implementação de unidades de ensino potencialmente } \\
\text { significativas sobre física térmica para alunos do } 2^{\circ} \text { ano do } \\
\text { ensino médio }\end{array}$ & 2015 & Dissertação \\
\hline
\end{tabular}

Quadro 3. Trabalhos de teses e dissertações analisados.

Os eventos elegidos para apreciação foram: Encontro de Físicos do Norte e Nordeste (EFNNE), Simpósio Nacional de Ensino de Física (SNEF), Encontro Nacional de Pesquisa em Educação em Ciências (ENPEC) e o Encontro de Pesquisa em Ensino de Física (EPEF). Pelo fato de serem muitas as edições dos eventos e, além disso, cada edição contar com um número grande de trabalhos, a seleção ocorreu inicialmente, somente mediante busca das palavras-chave nos títulos dos trabalhos apresentados na forma de pôster e comunicações orais, da seção de ensino de físicaiii e posterior leitura exploratória e analítica de 23 deles. O Quadro 4 mostra a relação dos 10 trabalhos que mais convergem com nossos pressupostos, juntamente com a autoria, ano e evento de publicação.

\begin{tabular}{|c|l|c|c|}
\hline \multicolumn{1}{|c|}{ Autor } & \multicolumn{1}{|c|}{ Título } & Ano & Publicação \\
\hline Weiller, et al. & $\begin{array}{l}\text { Construindo um coletor solar de baixo custo: uma } \\
\text { oportunidade para ensinar Física }\end{array}$ & 2007 & SNEF \\
\hline Bolfe \& Barlette & $\begin{array}{l}\text { Ensino de conceitos de física térmica a partir de situações: } \\
\text { uma aproximação aos invariantes operatórios de Vergnaud }\end{array}$ & 2009 & ENPEC \\
\hline
\end{tabular}




\begin{tabular}{|l|l|c|c|}
\hline Buss \& Nogueira & $\begin{array}{l}\text { Construção de um aquecedor solar de baixo custo: um } \\
\text { projeto de ensino e de aprendizagem para alunos do 10 ano } \\
\text { do ensino médio }\end{array}$ & 2011 & SNEF \\
\hline Oliveira, et al. & $\begin{array}{l}\text { Atividades de conceitualização em física térmica: buscando } \\
\text { invariantes operatórios }\end{array}$ & 2012 & EPEF \\
\hline Lima \& Amorin & $\begin{array}{l}\text { Conforto térmico em residências como uma proposta de } \\
\text { contextualização para o ensino de termodinâmica no ensino } \\
\text { médio }\end{array}$ & 2013 & EFNNE \\
\hline Lima \& Amorin & $\begin{array}{l}\text { Conforto térmico em residências como uma proposta de } \\
\text { contextualização para o ensino de termodinâmica no ensino } \\
\text { médio }\end{array}$ & 2013 & SNEF \\
\hline Lopes, et al. & $\begin{array}{l}\text { Sistema de aquecimento solar como tema de formação em } \\
\text { física/cidadania }\end{array}$ & 2013 & SNEF \\
\hline Menezes & $\begin{array}{l}\text { Aquecedor solar: uma possibilidade de ensino de física } \\
\text { através de temas geradores }\end{array}$ & 2013 & SNEF \\
\hline $\begin{array}{l}\text { Soares Neto \& } \\
\text { Leite }\end{array}$ & $\begin{array}{l}\text { Controle de temperatura a partir da construção da manta } \\
\text { térmica com caixas de leite do tipo longa vida (tetra pak) }\end{array}$ & 2014 & EFNNE \\
\hline Torcate, et al. & $\begin{array}{l}\text { Utilização de um aquecedor solar como ferramenta didática } \\
\text { para o ensino dos conceitos de calor }\end{array}$ & 2015 & SNEF \\
\hline
\end{tabular}

Quadro 4. Trabalhos analisados dos eventos científicos.

Norteamos a revisão da literatura, com os recortes escolhidos baseados em Laville \& Dionne (1999) que apontam que esta etapa representa um decurso crítico relacionado ao nosso objetivo, de forma a filtrar os trabalhos que nos despertam maior interesse e que contribuem de forma significativa com nossos propósitos, tendo, assim, a nossa escolha e interpretação.

Essa pesquisa é de cunho qualitativo porque estamos interessados em aspectos qualitativos dos trabalhos, que nos permitam diferenciá-los em termos de teorias defendidas, metodologias seguidas ou implicações para o ensino. Sendo assim, a nossa análise é essencialmente descritiva, apresentando aspectos que consideramos importantes, sendo fiéis à visão dos autores.

\section{RESULTADOS}

A seguir apresentaremos a descrição e análise dos materiais selecionados.

\subsection{Trabalhos encontrados em periódicos científicos}

Dos 41 trabalhos pré-selecionados, apresentamos uma breve descrição de alguns deles que corroboram com o foco da nossa revisão e que foram categorizados da seguinte forma:

\section{(i) Utilização de fornos/aquecedores solares no ensino da física térmica}

Dorado \& Rivera (2010) apresentam o resultado de uma experiência na qual elaboraram e expuseram diversos experimentos construídos com materiais de baixo custo, sendo que um desses foi uma estufa solar, construída com uma antena parabólica recoberta com papel alumínio. Os autores destacam que a divulgação e educação científica fora da sala de aula promove uma curiosidade científica e tecnológica, despertando o interesse dos alunos. 
Também se referindo a aquecedores solares (dispositivos análogos ao forno solar), mas num contexto de trabalho interdisciplinar, Dworarowski, et al. (2010) orientaram a construção de um aquecedor utilizando garrafas pet e caixas de leite para o desenvolvimento de conceitos relativos à hidrostática e à física térmica. Os autores destacam um trabalho interdisciplinar mais significativo com a área das ciências da natureza, um melhor aproveitamento dos alunos nas matérias relacionadas ao aquecedor e maior interação da escola com a comunidade.

Igualmente, Damasio \& Steffani (2007) descrevem um projeto interdisciplinar com vistas à discussão da diminuição do aquecimento global, a partir de um aquecedor de água caseiro. No desenvolvimento dos conceitos da física térmica, os alunos foram instigados a explicar cada etapa da construção do aquecedor, justificando a escolha dos materiais para cada parte do mesmo. Esse trabalho nos motiva a estruturar uma investigação sobre forno solar a partir da criação (pelos alunos) de um roteiro que descreva a funcionalidade do dispositivo a partir da seleção de materiais diferentes, por eles escolhidos, a partir da pesquisa sobre suas propriedades.

\section{(ii) Discussão histórica dos conceitos de calor e temperatura}

Pérez (2013) realiza uma descrição da evolução histórica da termometria, ressaltando os experimentos realizados para projetar os instrumentos, estabelecer os pontos fixos ou calibrar as escalas de medida. Apresenta também uma série de propostas de atividades experimentais que podem elucidar 0 desenvolvimento dos termômetros. Destaca ainda, que essas atividades podem ser realizadas em vários contextos, reforçando a necessária atenção à física experimental.

Hernández \& Dávalos (2012) destacam um marco teórico sobre as contribuições científicas que motivaram as transformações ocorridas no sistema de produção e as contribuições da termodinâmica para o surgimento da revolução industrial. Essas discussões são importantes para a sala de aula, como forma de mostrar aos estudantes o desenvolvimento da ciência e da tecnologia e seus efeitos na sociedade, bem como refletir sobre as mudanças ocorridas na forma do ensino.

Já Silva, et al. (2013) fazem uma síntese sobre a construção do conceito de calor até o final do século XVIII, apresentando exemplos sobre algumas visões deformadas que existem sobre as ciências, defendendo a não linearidade do avanço e progresso científico e a inexistência de verdades absolutas. Os autores propõem a inserção, na educação básica e/ou na formação inicial e continuada de professores, da história da termodinâmica.

\section{(iii) Confusão conceitual entre calor e temperatura}

Em se tratando da dificuldade encontrada sobre a conceituação do calor e da temperatura, Gomes (2012) analisou as teorias sobre calor a partir dos processos que envolvem aquecimento e resfriamento, e conclui que, embora a teoria do calórico não seja aceita cientificamente, os livros textos apresentam calor como uma grandeza que um corpo pode ganhar ou perder, tal qual a representação que os alunos fazem desse conceito. De maneira similar, Silva, et al. (2008) fornecem algumas reflexões sobre o conceito de calor e sua utilização na sala de aula. Os autores afirmam que as dificuldades em ensinar o conceito aparecem tanto nos livros quanto nas concepções dos professores.

Argumentando que, particularmente sobre a física térmica, os alunos não utilizam os conceitos de forma precisa, embora esse tema faça parte da vida cotidiana dos mesmos, Ramos, et al. (2012) 
investigaram se a pesquisa dirigida favorece um melhor aprendizado dos conceitos de calor (e sua transmissão) e temperatura, tendo uma turma de controle. Os autores destacam que essa forma de ensino mostrou uma diferença significativa do conhecimento conceitual adquirido em relação aos alunos que tiveram aula tradicional.

\section{(iv) Uso do referencial teórico de Vergnaud}

Baseados na teoria dos campos conceituais, Carvalho Jr. \& Aguiar Jr. (2008) investigaram a efetividade da utilização de várias estratégias e atividades através de uma sequência didática, elaborada a partir de diferentes situações. Como resultado, destacam que os alunos evoluíram conceitualmente quando comparados com a identificação de seus conhecimentos prévios.

Grings, et al. (2006) apresentam uma análise das dificuldades apresentadas por alunos sobre os tópicos do campo conceitual da termodinâmica, indicando possíveis invariantes operatórios que os mesmos utilizam nas diferentes situações e que estavam funcionando como obstáculo cognitivo para a aprendizagem do campo conceitual pesquisado. Em outro trabalho, Grings, et al. (2008) apresentam resultados de uma pesquisa semelhante, com a diferença que a investigação ocorreu com alunos que tinham estudado termodinâmica no ano anterior. Apesar disso, os autores destacam que os estudantes continuavam não compreendendo adequadamente os signos utilizados no referido campo conceitual.

Da leitura do material analisado, percebe-se que os conceitos da física térmica aparecem em contextos de tentativas de trabalho interdisciplinar, objetivando modificar o currículo compartimentalizado e descontextualizado que é desenvolvido na maior parte das escolas. Os conceitos de calor e temperatura são tratados como sinônimos pelos alunos, o que pode ser reflexo da forma como nos expressamos ou como os livros didáticos os tratam. O referencial teórico de Vergnaud é um bom suporte para a ocorrência de aprendizagem significativa, seja através da utilização de situações ou de identificação de possíveis invariantes operatórios. A discussão de aspectos históricos da construção do conceito de calor é importante porque resgata a visão de que a ciência não é neutra, linear e definitiva.

\subsection{Trabalhos encontrados em teses e dissertações}

Dos 40 trabalhos pré-selecionados, preocupamo-nos em fazer uma leitura analítica daqueles que discorriam sobre os conceitos da física térmica na educação básica, uma vez que alguns tinham enfoque no ensino superior e/ou formação continuada de professores, embora saibamos que esses aspectos também são importantes, pois são potenciais para refletir em melhorias no ensino. Os trabalhos analisados com maior ênfase podem ser classificados da seguinte forma:

\section{(i) Uso do referencial teórico de Ausubel}

Se a aprendizagem ocorre de forma significativa, o aluno, quando solicitado, tem mais facilidade de recordar o conhecimento aprendido. Buscando identificar o que os estudantes retomam das aulas de física do ano anterior, Pereira (2014) procurou compreender os processos que levam a essas retomadas, utilizando atividades investigativas sobre calor e temperatura. Aproximadamente um ano depois, foram aplicadas para os mesmos alunos atividades diferentes, mas que solicitavam a retomada do que tinha sido ensinado previamente. A autora destaca que os estudantes retomaram, 
além de conhecimentos e habilidades referentes ao ensino por investigação, atividades realizadas nas aulas, sentimentos e sensações.

Sobre a utilização de mapas conceituais no ensino, que é um instrumento facilitador na busca de evidências de aprendizagem significativa, Silva (2007) buscou verificar se a utilização desse instrumento é uma estratégia eficiente para promover e avaliar a aprendizagem significativa dos conceitos da calorimetria. As aulas se desenvolveram através de exposições orais acompanhadas de discussões e/ou atividades experimentais. Foram comparados os conhecimentos dos alunos através dos mapas conceituais que os mesmos construíram no decorrer das intervenções. Alguns pontos revelados pelo autor são que os indícios da aprendizagem significativa ficaram abaixo do esperado, embora tenha percebido uma evolução conceitual pelo fato dos alunos terem conseguido fazer algumas relações cruzadas entre os conceitos, o que indica a formação de proposições importantes.

\section{(ii) Contexto de formação técnica}

Poglia (2013) apresenta uma proposta de ensino de física térmica contextualizada e integrada à formação técnica. Para o desenvolvimento da pesquisa, foi utilizado um sistema de refrigeração doméstico montado sob a forma de uma bancada didática, associado com um texto de apoio. A partir desta proposta, houve um crescimento nos índices de acertos demonstrados pelos questionários de pré e pós-testes, além do elevado grau de satisfação dos alunos, o que pode sugerir que os alunos dedicam-se mais quando estão motivados, quando o material a ser estudado é potencialmente significativo e quando ocorre a integração das áreas técnica e básica.

Na dissertação de cunho contextualizado entre escola e trabalho, Dal Moro (2012), investigando alunos dos cursos técnicos em edificações e eletromecânica, na modalidade Educação de Jovens e Adultos (EJA), buscou identificar e estabelecer as relações entre os saberes, especialmente os da física, que os estudantes adquirem no trabalho e aqueles de cunho científico e teórico, aprendidos no ambiente escolar. $\mathrm{O}$ autor destaca que os trabalhadores buscam os saberes essenciais para a profissão em cursos ou em conversas com trabalhadores de maior experiência; os saberes de cunho prático e teórico se complementam de forma articulada, conferindo assim, mais autonomia nas suas funções, além de uma maior valorização profissional.

\section{Experimentação no ensino da física}

A partir da metodologia investigativa, Oliveira (2013) propôs a construção, com a utilização de materiais caseiros, de um termômetro de coluna líquida para desenvolver os tópicos da termologia. Os materiais utilizados para a análise foram questionários de cunho conceitual e experimental, bem como relatórios das atividades práticas. Alguns resultados apresentados pelo autor são que os alunos apresentaram dificuldade para expressar suas ideias e suas conclusões na forma escrita; apresentaram certa rejeição em fazer uso de ferramentas matemáticas durante o trabalho; a realização da atividade não possibilitou o mesmo nível de apropriação dos conceitos a todos os alunos, porém os mesmos passaram a participar da aula mais ativamente.

Já Michelena (2008) propôs atividades experimentais realizadas com materiais de baixo custo, além de exercícios conceituais. Os critérios utilizados para a avaliação da aprendizagem foram participação e envolvimento nas aulas e a construção de um mapa conceitual, além de uma prova sobre os conceitos abordados. Como resultado, a autora destaca que alguns alunos preferiram aprender física 
com a resolução de exercícios numéricos em vez de conceituais, embora não dispensassem a aula experimental.

A partir das concepções alternativas dos alunos sobre calor e temperatura, Rafael (2007) elaborou e aplicou uma estratégia de ensino constituída por atividades envolvendo história da ciência, bem como experimentos, a partir de uma proposta do livro do Grupo de Reelaboração do Ensino de Física (GREF). Os temas abordados foram o desenvolvimento da termodinâmica e a evolução dos conceitos de calor e de temperatura. A estratégia resultou em ganhos em termos da aprendizagem, promovendo a superação de algumas concepções iniciais dos estudantes.

Sias (2006) analisou a contribuição de atividades experimentais utilizando aquisição automática de dados. Alguns resultados destacados são que os alunos ficavam empolgados nas aulas em que faziam atividades experimentais, apresentaram melhora no desempenho no pós-teste realizado depois do desenvolvimento das atividades, e a metodologia favoreceu a ocorrência de aprendizagem significativa.

\section{(iii) Uso do referencial teórico de Vergnaud}

Sobre o ensino da física tendo como enfoque as situações, à luz da teoria de Vergnaud, Amaral (2010) elaborou uma estratégia de ensino baseada na atividade prática cuja realização se deu por meio de resoluções de situações-problema, visando a aprendizagem significativa dos processos de transmissão de energia térmica. Antes da aplicação das situações-problema, foi aplicado um teste, buscando a identificação de subsunçores. Como resultados, a autora destaca que nas duas turmas houve motivação de grande parte dos alunos; que a turma de controle teve um comportamento mais adequado durante as aulas, se mostrando mais aplicada e que os alunos que menos se envolveram nas discussões não tiveram nenhuma evolução conceitual. Uma consideração importante sobre estes resultados é que, por mais que o material a ser ensinado seja potencialmente significativo, a aprendizagem significativa depende da pré-disposição dos alunos.

Tendo como foco a teoria dos campos conceituais desenvolvida através de Unidades de Ensino Potencialmente Significativas (UEPS ${ }^{\mathrm{iv}}$ ), Pradella (2014) analisou a aplicação de quatro dessas unidades sobre conceitos da física térmica. A metodologia utilizada conteve simulações interativas, aulas expositivo-dialogadas, resolução de exercícios, realização de avaliações, construção e apresentação de mapas conceituais. Com o desenvolvimento das atividades não houve avaliação negativa em relação à organização das aulas ou à proposição da abordagem através das UEPS; no entanto, alguns alunos demonstraram preocupação em relação à quantidade de exercícios propostos. Sobre a avaliação do aprendizado, os alunos tiveram maior clareza em relação aos conceitos abordados nas unidades de ensino.

Trabalho que também utiliza a estratégia didática das UEPS foi realizado por Faccin (2015). Uma das UEPS abarcou o conceito de temperatura, e outra, o conceito de calor. Como resultados da pesquisa, a autora destaca que as Unidades de Ensino Potencialmente Significativas apontaram indícios de aprendizagem significativa, haja vista que foram estruturadas de forma a possibilitarem a evolução conceitual dos alunos, a partir de questionamentos que se tornavam mais complexos conforme os alunos progrediam no campo conceitual.

Das dissertações e teses analisadas, percebe-se um desejo de discutir os conceitos da física térmica em um contexto de formação técnica, como forma de dar significado a esses conceitos em uma de 
formação profissional - foco do nosso trabalho docente, que busca propiciar momentos de integração curricular entre a física e à área técnica. As teorias de Ausubel e Vergnaud fundamentam diversos trabalhos (inclusive o nosso), por serem teorias complementares e que buscam avanços no processo de ensino-aprendizagem. Alguns trabalhos fazem um comparativo entre duas turmas, com uma delas sendo controle, o que não nos impulsiona a seguir o mesmo caminho, por acreditarmos que não é possível comparar sujeitos diferentes em realidades didáticas desiguais. A experimentação é uma estratégia importante no ensino da física porque possibilita diversos níveis de investigação, facilitando a aprendizagem de conceitos que explicam e/ou justificam fenômenos físicos.

\subsection{Trabalhos encontrados em eventos científicos}

Os trabalhos analisados dos eventos científicos podem ser categorizados da seguinte forma:

\section{(i) Ensino contextualizado da física térmica}

Relacionando o conforto térmico com a capacidade de o organismo humano realizar trabalho, Lima \& Amorim (2013) descrevem um roteiro para analisar o nível de conforto com as pessoas desempenhando atividades cotidianas, a partir da coleta de dados de temperatura e umidade relativa do ar, tanto na escola quanto na casa dos alunos. Além disso, os autores propuseram uma solução para reduzir o desconforto domiciliar. Para isso, construíram dois protótipos de casa, com telhados de cor diferente - um com telhado branco e outro com telhado cinza. Como resultados, os autores destacam que todos os sujeitos, alunos e familiares se envolveram fortemente com o projeto; o trabalho foi desenvolvido inteiramente no horário regular da aula e foi possível desenvolvê-lo de forma contextualizada com a realidade dos alunos.

Em outro trabalho Lima \& Amorim (2013b) relatam que a temperatura atingida pelo telhado pintado de branco foi $5^{\circ} \mathrm{C}$ mais baixa que do telhado pintado de cinza, quando ambos foram expostos por $24 \mathrm{~h}$ à radiação. Na nossa pesquisa, aspectos relacionados às propriedades dos materiais utilizados para o forno solar devem favorecer que o forno tenha uma alta eficiência e atinja temperaturas elevadas no seu interior. $\mathrm{E}$ uma das propriedades é a cor escolhida.

Objetivando mostrar aos alunos a viabilidade e eficácia das mantas térmicas feitas com caixas de leite longa vida no conforto térmico em residências, Soares Neto \& Leite (2014) construíram dois protótipos de casa, ambas cobertas com cimento-amianto e também uma manta térmica construída a partir da junção de várias caixas tetra pak. A superfície interna do telhado de uma das casas foi coberta com a manta. As medidas de temperatura, meia hora após a exposição à radiação, indicaram uma diferença de temperatura de $8^{\circ} \mathrm{C}$ entre as casas, o que demonstrou a eficiência da manta térmica.

\section{(ii) Utilização de fornos/aquecedores solares no ensino da física térmica}

A partir da constatação de que pessoas carentes que têm aquecedores solar em casa para reduzir os gastos com energia, por exemplo, desconhecem o funcionamento desses sistemas bem como a forma de utilização adequada para obter economia na conta de energia elétrica, Lopes et al., (2013) descrevem o resultado de um trabalho desenvolvido a partir do tema gerador "aquecimento solar". Um grupo de alunos, em encontros extraclasse, retomou os conceitos aprendidos anteriormente sobre física térmica para tentar explicar o funcionamento do aquecedor solar. O estudo dos conceitos da transmissão do calor, tendo o coletor como tema gerador, proporcionou maior envolvimento dos estudantes e contribuiu para a aprendizagem de conhecimentos e fenômenos físicos, o que pode 
resultar na melhoria da qualidade de vida dos sujeitos participantes, bem como daquelas pessoas que vivem no seu entorno, pois os alunos se tornaram disseminadores da utilização correta desses dispositivos.

Menezes (2013), embasado no referencial teórico dos temas geradores e dos três momentos pedagógicos, propôs a construção de um aquecedor solar de baixo custo. A partir de uma pesquisa realizada pelos estudantes sobre construção e funcionamento desses dispositivos, diversos conceitos da física térmica foram estudados. A partir do conhecimento sistematizado, os alunos construíram um sistema para aquecimento de água utilizando garrafas pet, embalagens de leite longa vida e canos de PVC. Cada grupo apresentou os resultados do seu aquecedor através de um seminário realizado para os colegas e professores. O autor destaca que o trabalho possibilitou a elucidação dos conhecimentos prévios dos estudantes para a posterior construção do conhecimento científico, além de os alunos terem tido maior facilidade de aprender os conceitos físicos depois da realização da pesquisa.

Buss \& Nogueira (2011) apresentam o resultado de uma experiência didática na qual foram estudados os conceitos relativos à física térmica e à ondulatória e que culminou com a construção de um aquecedor de água de baixo custo utilizando energia solar. Algumas aulas constituíram-se de demonstração de aspectos teóricos e resolução de exercícios referentes aos conteúdos; outras, foram desenvolvidas através da exposição de vídeos e pequenos experimentos; por fim, também tiveram algumas aulas destinadas à confecção do aquecedor solar. Como resultado, os autores afirmam que houve evidências de aprendizagem significativa, de modo que os novos conhecimentos foram ancorados na estrutura cognitiva existente dos sujeitos.

Também para discutir os conceitos da física térmica, Weiller et al. (2007) propuseram a construção de um coletor solar de baixo custo. Por dois dias o coletor foi exposto à radiação, do início da manhã ao início da noite e nesses dias, embora as condições climáticas tenham sido diferentes, a temperatura final da água sofreu uma variação de aproximadamente $16^{\circ} \mathrm{C}$.

Com o objetivo de investigar as contribuições referentes ao desenvolvimento de um ensino de física contextualizado a partir da utilização de um aquecedor solar de baixo custo, Torcate et al. (2015) utilizaram os mapas conceituais como ferramenta para avaliação. Os tópicos abordados nas discussões foram os processos de propagação do calor. Foram construídos dois mapas conceituais: um com os conhecimentos prévios dos sujeitos sobre os conceitos abordados e o outro após a intervenção didática. Essa intervenção ocorreu durante duas aulas, nas quais os alunos discutiram os princípios físicos presentes no aquecimento da água no aquecedor, o qual foi levado pronto para a escola. Como resultado, os autores destacam que os alunos conseguiram ver o caráter contextualizado da física, além de terem conseguido estabelecer importantes relações conceituais através dos mapas.

\section{(iii) Uso do referencial teórico de Vergnaud}

Dois trabalhos se preocuparam em identificar possíveis invariantes operatórios referentes aos conceitos da física térmica. Oliveira et al. (2012) analisaram algumas situações nas quais uma estudante do ensino médio confronta suas concepções e representações da física térmica, com aquelas formulações teóricas da área. A partir das falas da aluna, foi possível perceber que ela difere 
calor de temperatura; reconhece calor como um processo de transferência de energia e, contudo, tem a ideia que se há calor, há variação de temperatura.

Sobre o conceito de dilatação térmica, Bolfe \& Barlette (2009) destacam que os indícios de possíveis invariantes operatórios mostraram dificuldades conceituais que podem servir de obstáculo epistemológico para a aprendizagem de novos conceitos. Os invariantes operatórios são importantes de serem investigados como forma de identificar o que os alunos têm internalizado sobre os conceitos, mas buscando possibilitar que os mesmos sejam enriquecidos e diferenciados.

Dos trabalhos analisados dos eventos científicos, a maioria deles relata experiências de estudo dos conceitos da física térmica a partir de fornos/aquecedores solares. Esses dispositivos são recursos didáticos potenciais para a discussão dos conceitos de calor (e sua transmissão), dilatação térmica, temperatura. Além da motivação para as discussões dos conceitos, promovem o debate crítico acerca da racionalização de energia e de condições de conforto térmico que propiciam melhoras nas condições de vida das pessoas. As concepções prévias dos alunos, como apontado nos trabalhos fundamentados em Vergnaud, representam obstáculos cognitivos para o avanço no campo conceitual da física térmica, em função da confusão conceitual existente, por exemplo, entre as definições de calor e temperatura.

\section{CONSIDERAÇÕES}

A revisão bibliográfica da nossa investigação não se finda com esta tessitura, uma vez que novas publicações são realizadas constantemente. Além disso, nem todos os textos selecionados foram descritos aqui, haja vista o demasiado número de trabalhos encontrados. Focamos nos trabalhos desenvolvidos com alunos do ensino médio, que abordassem conceitos da calorimetria, que utilizassem o forno/aquecedor solar como recurso didático, que visassem favorecer a aprendizagem significativa dos referidos conceitos, que trouxessem embasamento na teoria dos campos conceituais, que incluíssem a interdisciplinaridade como eixo central e que, na medida do possível, tivessem sido desenvolvidos em cursos técnicos - o que condiz com nossa realidade de trabalho. Levando isso em consideração, alguns apontamentos importantes de serem destacados a partir da leitura dos textos são:

Os aspectos históricos da calorimetria fundamentam nove trabalhos que buscam suscitar discussões sobre o desenvolvimento científico e tecnológico, com um viés crítico sobre suas implicações na sociedade. Além disso, auxiliam no entendimento da construção dos conceitos e podem justificar a existência de algumas concepções prévias, como por exemplo, que calor é uma energia contida em nos corpos.

O mapa conceitual constitui-se numa ferramenta potencial de identificação do conhecimento construído pelos estudantes, uma vez que através desse recurso é possível verificar como os sujeitos integram conceitos e proposições em suas estruturas cognitivas. Baseados nisso, Silva (2007), Pradella (2014) e Torcate et al. (2015) relatam experiências didáticas que contribuem para a aprendizagem significativa, verificada a partir do mapeamento conceitual.

O forno/aquecedor solar é um recurso didático promissor para a discussão da física térmica. Além disso, é um instrumento que favorece a interdisciplinaridade, por necessitar de discussões acerca de diversas disciplinas, que vão desde os conceitos físicos, até os de cunho social. Nos eventos científicos foram encontrados onze trabalhos relacionados com a construção e/ou utilização desses dispositivos 
no ensino de física, entre eles os de Damasio \& Steffani (2007), Weiller, et al. (2007), Dworarowski, et al. (2010), Dorado \& Rivera (2010) e Lopes, et al. (2013), o que ratifica a importância de sua construção.

A interdisciplinaridade é uma forma de romper com o currículo fragmentado, no qual os professores trabalham de forma isolada. Apostando no fazer interdisciplinar no ensino de ciências, destacam-se os trabalhos de Lima \& Amorim (2013; 2013b), que acreditam nos ganhos de se investir em um trabalho interdisciplinar, sempre defendendo o diálogo entre as disciplinas envolvidas, buscando transcender a multidisciplinaridade.

Um campo conceitual é complexo de ser aprendido e, sendo assim, é necessária a apresentação de diversos tipos de situações, de modo que o repertório de esquema dos estudantes se amplia conforme se aumenta a classe de situações (MOREIRA, 2009). Trabalhos como os de Grings, et al. (2006, 2008), Carvalho Jr. \& Aguiar Jr. (2008), Amaral (2010) e Oliveira et al. (2012) apontam que os conceitos intuitivos sobre calor e temperatura podem servir de obstáculos epistemológicos para a construção de novos conceitos. Para superar isso, os trabalhos destacam o papel mediador do professor, bem como a necessidade de diferentes abordagens para possibilitar que os alunos evoluam no campo conceitual da física térmica.

Também, um campo conceitual é complexo de ser aprendido, pois é constituído de caminhos com avanços e retrocessos. Como alguns trabalhos apontam, os alunos dificilmente, em um curto intervalo de tempo, substituirão o conceito de calor empregado no dia a dia como sinônimo de temperatura, mas entendemos que nosso papel enquanto docentes é fazer perceberem em que diferem e incentiválos a, pelo menos no espaço acadêmico, expressarem-se de forma correta.

Sobre as concepções alternativas carregadas pelos alunos, diversos trabalhos, tais como Ramos, et al. (2012), Rafael (2007) e Pradella (2014) defendem e avançam em relação a metodologias de ensino que favoreçam a aprendizagem significativa dos conceitos de calor e temperatura.

Trabalhos como os de Oliveira (2013), Michelena (2008), Rafael (2007) e Sias (2006) destacam a importância da experimentação no ensino da física térmica como forma de modificar as concepções prévias dos alunos sobre os conceitos de calor e temperatura.

Os conceitos da física térmica são fonte para diversos trabalhos realizados continuamente, o que, ao mesmo tempo em que remete à importância da discussão desses conceitos, levanta questionamentos sobre a difusão dessas pesquisas para outras realidades e contextos para promover também a aprendizagem significativa dos referidos conceitos.

\section{REFERÊNCIAS}

AMARAL, Ana Paula da Costa. A atividade prática abordada por meio de situações- problema, visando a promoção da aprendizagem significativa dos conceitos relacionados aos processos de transmissão da energia térmica, no ensino médio. Brasília: UnB, 2010. Dissertação, Instituto de Ciências Biológicas, Instituto de Física, Instituto de Química, Universidade de Brasília, 2010.

BOLFE, Lurdes Eliane Rothmund; BARLETTE, Vania Elisabeth. Ensino de conceitos de física térmica a partir de situações: uma aproximação aos invariantes operatórios de Vergnaud. In: ENCONTRO NACIONAL DE PESQUISA EM EDUCAÇÃO EM CIÊNCIAS, 7, 2009, Florianópolis. Atas... Florianópolis: Associação Brasileira de Pesquisa em Educação em Ciências, 2009. 
BUSS, Cristiano da Silva; NOGUEIRA, Christiano. Construção de um aquecedor solar de baixo custo: um projeto de ensino e de aprendizagem para alunos do $1^{\circ}$ ano do ensino médio. In: SIMPÓSIO NACIONAL DE ENSINO DE FÍSICA, 19, 2011, Manaus. Atas... São Paulo: Sociedade Brasileira de Física, 2011.

CARVALHO JÚNIOR, Gabriel Dias; AGUIAR JÚNIOR, Orlando. Os campos conceituais de Vergnaud como ferramenta para o planejamento didático. Caderno Brasileiro de Ensino de Física, Florianópolis, v. 25, n. 2, p. 207-227, 2008.

DAL MORO, Guilherme André. Do trabalho para a escola: olhares de trabalhadoresestudantes e professores sobre as relações entre o saber da prática e o saber da escola. Curitiba: UTFPR, 2012. Dissertação, Programa de Pós-Graduação em Tecnologia, Universidade Técnica federal do Paraná, 2012.

DAMASIO, Felipe; STEFFANI, Maria Helena. Ensinando física com consciência ecológica e com materiais descartáveis. Revista Brasileira de Ensino de Física, São Paulo, v. 29, n. 4, p.593 - 597, 2007.

DORADO, Remigio Cabral; RIVERA, José Luis Maldonado. Enseñanza de las ciencias físicas a estudiantes de primaria y secundaria por medio de sencillos talleres científicos. LatinAmerican Journal of Physics Education, México, v. 4, n. 2, p. 415-421, 2010.

DWORAKOWSKI, Luis Antonio de Quadros; MARRANGHELLO, Guilherme Frederico; DORNELES, Pedro Fernando Teixeira. $\mathbf{O}$ aquecedor solar na sala de aula. Experiências em Ensino de Ciências, Cuiabá, vol.5, n. 2, p. 147-162, 2010.

FACCIN, Franciele. Implementação de unidades de ensino potencialmente significativas sobre física térmica para alunos do $\mathbf{2}^{\circ}$ ano do ensino médio. Santa Maria: UFSM, 2015. Dissertação, Programa de Pós-Graduação em Ensino de Física, Universidade Federal de Santa Maria, 2015.

GOMES, Luciano Carvalhais. A ascenção e queda da teoria do calórico. Caderno Brasileiro de Ensino de Física, Florianópolis, v. 29, n. 3, p. 1030-1073, 2012.

GRINGS, Edi Terezinha de Oliveira; CABALLERO, Concesa; MOREIRA, Marco Antonio. Avanços e retrocessos dos alunos no campo conceitual da Termodinâmica. Revista Electrónica de Enseñanza de las Ciencias, Vigo, v. 7, n. 1, p. 23-46, 2008.

GRINGS, Edi Terezinha de Oliveira; CABALLERO, Concesa; MOREIRA, Marco Antonio. Possíveis indicadores de invariantes operatórios apresentados por estudantes em conceitos da termodinâmica. Revista Brasileira de Ensino de Física, São Paulo, v. 28, n. 4, p. 463-471, 2006.

HERNÁNDEZ, Alberto Francisco Sandino; DÁVALOS, Lilia Montiel. La Termodinámica como origen de la revolución industrial del siglo XVIII. Latin- American Journal of Physics Education, México, v. 6, n. 4, p. 652-654, 2012.

LIMA, Eduardo Couto; AMORIM, Helio Salim de. Conforto térmico em residências como uma proposta de contextualização para o ensino de termodinâmica no ensino médio. In: ENCONTRO DE FÍSICOS DO NORTE E NORDESTE, 31, 2013, Campina Grande. Atas... São Paulo: Sociedade Brasileira de Física, 2013.

LIMA, Eduardo Couto; AMORIM, Helio Salim de. Conforto térmico em residências como uma proposta de contextualização para o ensino de termodinâmica no ensino médio. In: 
SIMPÓSIO NACIONAL DE ENSINO DE FÍSICA, 20, 2013, São Paulo. Atas... São Paulo: Sociedade Brasileira de Física, 2013b.

LOPES, João Paulo; FERREIRA, Waldo Franco; AUTH, Milton A. Sistema de aquecimento solar como tema de formação em física/cidadania. In: SIMPÓSIO NACIONAL DE ENSINO DE FÍSICA, 20, 2013, São Paulo. Atas... São Paulo: Sociedade Brasileira de Física, 2013.

MENEZES, Marcos Vinícius Marcondes de. Aquecedor solar: uma possibilidade de ensino de física através de temas geradores. In: SIMPÓSIO NACIONAL DE ENSINO DE FÍSICA, 20, 2013, São Paulo. Atas... São Paulo: Sociedade Brasileira de Física, 2013.

MICHELENA, Juleane Boeira. Física térmica: uma abordagem histórica e experimental. Porto Alegre: UFRGS, 2008. Dissertação, Instituto de Física, Universidade Federal do Rio Grande do Sul, 2008.

OLIVEIRA, Cleidson Santiago. Atividade experimental investigativa: construção do termômetro de coluna líquida. São Carlos: UFSCAR, 2013. Dissertação, Centro de Ciências Exatas e Tecnologia, Universidade Federal de São Carlos, 2013.

OLIVEIRA, Vanessa Vale; TEODORO JÚNIOR, Roberto de Souza; SILVA, Luiz Gustavo; CARVALHO JÚNIOR, Gabriel Dias. Atividades de conceitualização em física térmica: buscando invariantes operatórios. In: ENCONTRO DE PESQUISA EM ENSINO DE FÍSICA, 14, 2012, Maresias. Atas... São Paulo: Sociedade Brasileira de Física, 2012.

PEREIRA, Marta Máximo. Memória mediada na aprendizagem de Física: problematizando a afirmação "Não me lembro de nada das aulas do ano passado!". São Paulo: USP, 2014. Tese, Instituto de Física, Instituto de Química, Instituto de Biociências, Universidade de São Paulo, 2014.

PÉREZ, Justo R. Perspectivas didácticas de una aproximación histórica a la medición y concepto de Temperatura. Latin American Journal of Physics Education, México, v. 7, n. 3, p. 456467, 2013.

POGLIA, Rodrigo. 0 refrigerador doméstico como instrumento motivador para o ensino de física térmica: uma proposta para o curso Técnico Integrado em Refrigeração e Climatização. Porto Alegre: UFRGS, 2013. Dissertação, Instituto de Física, Universidade Federal do Rio Grande do Sul, 2013.

PRADELLA, Marcos. Estudo de conceitos da termodinâmica no ensino médio por meio de UEPS. Porto Alegre: UFRGS, 2014. Dissertação, Instituto de Física, Universidade Federal do Rio Grande do Sul, 2014.

RAFAEL, Francisco Josélio. Elaboração e aplicação de uma estratégia de ensino sobre os conceitos de calor e de temperatura. Natal: UFRN, 2007. Dissertação, Centro de Ciências Exatas e da Terra, Universidade Federal do Rio Grande do Norte, 2007.

RAMOS, María de la Cruz Medina; ORTEGA, Alfredo López; MORA, César. An alternative for the teaching and learning of the heat transmission topic with base in the directed research for high-school students. Latin American Journal of Physics Education, México, v. 6, Suppl. I, p. 222-225, 2012.

SIAS, Denise Borges. A aquisição automática de dados proporcionando discussões conceituais na física térmica do ensino médio. Porto Alegre: UFRGS, 2006. Dissertação, Instituto de Física, Universidade Federal de Santa Maria, 2006. 
SILVA, Ana Paula Bispo; FORATO, Thaís Cyrino de Mello; GOMES, José Leandro de A. M. Costa. Concepções sobre a natureza do calor em diferentes contextos históricos. Caderno Brasileiro de Ensino de Física, Florianópolis, v. 30, n. 3, p. 492-537, 2013.

SILVA, Gilmar. Mapas conceituais como instrumentos de promoção e avaliação da aprendizagem significativa de conceitos de calorimetria, em nível médio. Brasília: UnB, 2007. Dissertação, Instituto de Física, Instituto de Química, Universidade de Brasília, 2007.

SILVA, Osmar Henrique Moura; LABURÚ, Carlos Eduardo; NARDI, Roberto. Reflexões para subsidiar discussões sobreo conceito de calor na sala de aula. Caderno Brasileiro de Ensino de Física, Florianópolis, v. 25, n. 3, p. 383-396, 2008.

SOARES NETO, João Augusto; LEITE, Tarso Leandro Holanda. Controle de temperatura a partir da construção da manta térmica com caixas de leite do tipo longa vida (tetra pak). In: ENCONTRO DE FÍSICOS DO NORTE E NORDESTE, 32, 2014, João Pessoa. Atas... São Paulo: Sociedade Brasileira de Física, 2014.

TORCATE, Ariela Sarmento; CINTRA JÚNIOR, José Ancelmo da S.; SILVA, Alexandre Valença do Nascimento; LIMA, José Roberto Tavares de. Utilização de um aquecedor solar como ferramenta didática para o ensino dos conceitos de calor. In: SIMPÓSIO NACIONAL DE ENSINO DE FÍSICA, 21, 2015, Uberlândia. Atas... São Paulo: Sociedade Brasileira de Física, 2015.

WEILLER, Luiz Artur; ANDRADE, Nylton Gomes; MOURA, Thiago Daniel; ALMEIDA, Teddy; CORREA, Rodrigo Izidoro; DICKMAN, Adriana Gomes. Construindo um coletor solar de baixo custo: uma oportunidade para ensinar Física. In: SIMPÓSIO NACIONAL DE ENSINO DE FÍSICA, 17, 2007, Manaus. Atas... São Paulo: Sociedade Brasileira de Física, 2007.

Referências gerais

AUSUBEL, David Paul. Aquisição e retenção de conhecimentos: uma perspectiva cognitiva. Tradução Lígia Teopisto. 1. ed. Portugal: Editora Plátano, 2003.

FAZENDA, Ivani Catarina Arantes. Interdisciplinaridade-transdisciplinaridade: visões culturais e epistemológicas. In: FAZENDA, Ivani Catarina Arantes (Org.). $O$ que é interdisciplinaridade? São Paulo: Cortez, 2008.

FAZENDA, Ivani Catarina Arantes. Integração e interdisciplinaridade no ensino brasileiro: efetividade ou ideologia. 6. ed. São Paulo: Edições Loyola, 2011.

GIL, Antônio Carlos. Como elaborar projetos de pesquisa. 4. ed. São Paulo: Atlas, 2002.

HALLIDAY, David; RESNICK, Robert; WALKER, Jearl. Fundamentos de física II: gravitação, ondas e termodinâmica. Tradução de Ronaldo Sérgio de Biasi. Rio de Janeiro: LTC, 2009.

LAVILLE, Christian; DIONNE, Jean. A construção do saber: manual de metodologia da pesquisa em ciências humanas. Tradução de Heloisa Monteiro e Francisco Settineri. Porto Alegre: Artmed, 1999.

LOPES, Alice Ribeiro Casimiro. Conhecimento escolar: ciência e cotidiano. Rio de Janeiro: Uderj, 1999.

MARKONI, Marina de Andrade. LAKATOS, Eva Maria. Metodologia do trabalho científico: procedimentos básicos, pesquisa bibliográfica, projeto e relatório, publicações e trabalhos científicos. 7. ed. São Paulo: Atlas, 2011. 
MOREIRA, Marco Antonio. Teorias de Aprendizagem. São Paulo: EPU, 1999.

POMBO, Olga. Epistemología de la interdisciplinariedad: la construcción de un nuevo modelo de comprensión. INTERdisciplina, v. 1, n.1, p. 21-50, 2013.

VERGNAUD, Gerard. Psicologia do desenvolvimento cognitivo e didáctica das matemáticas. Um exemplo: as estruturas aditivas. Análise Psicológica, Lisboa, v.1, n. 5, p. 75-90, 1986.

VERGNAUD, Gerard. Conceptual development and learning. Revista Qurriculum, Laguna, n. 26, p. 39-59, 2013.

YOUNG, Hugh D.; FREEDMAN, Roger A. Física II: termodinâmica e ondas. Tradução de Sonia Midori Yamamoto. São Paulo: Addison Wesley, 2009.

\footnotetext{
Informações obtidas através de pesquisa realizada no endereço eletrônico < https://sucupira.capes.gov.br/sucupira/public/consultas/coleta/veiculoPublicacaoQualis/listaConsultaGeralPeriodicos.jsf>, no dia 23/05/2017.

ii Em nossa busca não fizemos distinção entre os programas de mestrado acadêmico e profissional.

iii Eventos como o Encontro de Físicos do Norte e Nordeste apresenta programação diferente para a área de ensino de física.

iv Sequências de ensino fundamentadas teoricamente, voltadas para a aprendizagem significativa que podem estimular a pesquisa aplicada em ensino, aquela voltada diretamente à sala de aula.
} 